\title{
Having a Primary Care Provider is the Strongest Predictor of Successful Follow-up of Participants in a Clinical Trial
}

\author{
Samuel H. Friedman, MD, Chinazo O. Cunningham, MD, MS, Juan Lin, PhD, \\ Linda B. Haramati, MD, MS, and Jeffrey M. Levsky, MD, PhD
}

Purpose: Ethnic minorities, women, and those of low socioeconomic status are widely underrepresented in clinical trials. Few studies have explored factors associated with successful follow-up in these historically difficult-to-reach patients. This study's objective was to identify patient characteristics and methods of contact that predict successful contact for follow-up in an urban, predominantly ethnic minority, majority-women, poor population to help devise strategies to improve retention.

Methods: We retrospectively reviewed records from a prospective randomized control trial of 400 hospitalized chest pain patients to determine which characteristics were associated with successful telephone follow-up at 1 year after enrollment. We assessed demographic variables, medical history, and social factors by using bivariate analyses. A multivariate analysis was performed using variables from the bivariate analysis with $P \leq .2$.

Results: The overall successful 1-year follow-up rate was $95 \%(381 / 400)$. Study participants who completed follow-up were significantly more likely to have a primary care physician (PCP) (88\% [337/ $381]$ versus 68\% [13/19]), speak English natively (52\% [199/381] versus 26\% [5/19]), have a higher Charlson comorbidity index score, and identify as women (64.0\% [244/381] versus $42.1 \%$ [8/19]). Having a PCP and native English language remained significant at multivariate analysis. Socioeconomic status score, quantity of contact information recorded at recruitment, and insurance status were not significantly associated with successful follow-up.

Conclusions: Patients engaged with the health care system by having a PCP are significantly more likely to achieve follow-up. Successful follow-up is also associated with native English speaking. The potential of improving follow-up by facilitating connections with health care providers requires further study. (J Am Board Fam Med 2020;33:431-439.)

Keywords: Ethnic Groups, Minority Groups, Retrospective Studies

\section{Introduction}

Clinical research informs decision making and provides the foundation for evidence-based medicine and best practices. ${ }^{1}$ Comparative effectiveness

\footnotetext{
This article was externally peer reviewed.

Submitted 20 January 2019; revised 2 February 2020; accepted 5 February 2020.

From Department of Radiology, Montefiore Medical Center and Albert Einstein College of Medicine, Bronx, NY (SHF, LBH, JML); Department of Family and Social Medicine, Montefiore Medical Center and Albert Einstein College of Medicine, Bronx, NY (COC); Department of Medicine, Montefiore Medical Center and Albert Einstein College of Medicine, Bronx, NY (COC, LBH, JML); Department of Epidemiology \& Population Health, Montefiore Medical Center and Albert Einstein College of Medicine, Bronx, NY (JL); Current Address: Department of Family Medicine, Overlook Medical Center, Summit, NJ (SHF).
}

research with randomized clinical trials is a preferred method for testing an intervention for a particular population or setting. ${ }^{2}$ This helps clinicians to maximize outcomes, knowing that the evidence they are using is specific for the task at hand and tested under comparable conditions.

The length of follow-up and retention are particularly important, as truncated follow-up can erroneously indicate that an intervention is more or

Acknowledgements/Funding: Funding of primary trial (PROSPECT): American Heart Association Clinical Research Program Grant 0885024D.

Conflicting and Competing Interests: None declared.

Corresponding author: Samuel H. Friedman, MD, 183 Route 206, Suite 1, Flanders, NJ 07836 (E-mail: samuel. friedman@med.einstein.yu.edu). 
less efficacious than it would be with long-term, more realistic use. ${ }^{3,4}$ A significant challenge in clinical research is keeping participants within a study for an extended period. Loss of research subjects in follow-up occurs for numerous reasons. ${ }^{3,5}$ It is useful to understand why this happens so that efforts can be made to mitigate that potential loss. ${ }^{6}$

It is well documented that racial/ethnic minorities are underrepresented in clinical trials ${ }^{7,8}$ and, in particular, cardiovascular studies. Some of the oftencited reasons include a distrust of medical research, economic burdens, and poor access to care. ${ }^{8,9}$ With regard to cardiovascular health in general, racial/ ethnic minorities have both a higher prevalence of disease and worse outcomes. ${ }^{10}$ These inequalities are particularly problematic because the existing literature provides suboptimal guidance for the treatment of some patients. Women are widely underrepresented within clinical trials. ${ }^{11-13}$ The dominance of white males in cardiovascular clinical trials has resulted in data that, in many cases, apply to less than half of the population. As a result, funding organizations have placed renewed emphasis on the explicit study of minority populations and women. ${ }^{14}$

Some of the challenges involved in performing the multicultural and gender-balanced clinical research needed to inform practice are already apparent. Patient characteristics, including male gender, ${ }^{15}$ black race, ${ }^{16}$ and young children in the household ${ }^{17}$ are associated with reduced retention rates in clinical trials. Patient involvement in the medical system and language preferences are potential determinants of success in follow-up. Different methods of patient recruitment, community involvement, and cultural tailoring have been studied to improve trial retention. ${ }^{18}$ Identifying correlates of poor follow-up allows a specific direction of efforts to retain at-risk patients and appropriate allocation of study resources.

The purpose of the present study is to assess the factors associated with successful 1-year follow-up from a multiethnic, majority-women, urban, cardiovascular randomized controlled trial to inform strategies to improve patient retention.

\section{Methods}

The Clinical Trial is registered at https://clinicaltrials. gov/ct2/show/NCT00705458 under ClinicalTrials. gov Identifier NCT00705458.

\section{Study Design}

This study is a secondary analysis of the PROSPECT trial, which was conducted between July 2008 and December 2013. ${ }^{19,20}$ PROSPECT was a randomized controlled trial of 400 intermediate-risk chest pain patients admitted to telemetry at Montefiore Medical Center in the Norwood section of Bronx, NY. There were no recruitment restrictions regarding gender, race/ethnicity, native language, or health insurance. Participants were randomized to receive coronary computed tomography or radionuclide stress myocardial perfusion imaging, with the primary outcome variable being cardiac catheterization not leading to revascularization within 1 year. There was no statistically significant difference between the 2 imaging modalities with regard to the primary outcome. The trial design and primary outcome variable required 1 year of follow-up after recruitment.

\section{Enrollment and Follow-up}

Enrollment occurred between July 2008 and March 2012. At the time of enrollment, patients were asked for multiple phone numbers and the name and phone number of a relative or other close contact. Patients were called for telephone questionnaires at 6 and 12 months after enrollment to discover subsequent cardiac events and hospitalizations. If a patient could not be reached by telephone after at least 4 attempts either directly or via a close contact, further attempts were made through a primary care physician (PCP) or cardiologist identified from electronic medical records. Providers and facilities outside of Montefiore were contacted by telephone for records when patients provided this information and their permission. Additionally, continuous telephone and electronic (ie, e-mail and texting) follow-up after 1 year was conducted in a chronological fashion based on enrollment date. At the end of the study period, patients were contacted again to determine whether they experienced major adverse cardiovascular events.

\section{Follow-up Outcome and Variables of Interest}

The current study's primary outcome variable was defined as successful contact with the patient at or beyond 12 months after enrollment. This exploratory analysis assessed potential predictor variables, including sociodemographic data (age; race/ethnicity; gender; native or nonnative English speaker; socioeconomic status (SES) score based on 
Table 1. Patient Baseline Characteristics $(n=400)$

\begin{tabular}{|c|c|}
\hline Characteristic & Values* \\
\hline Age (y) & $57 \pm 11$ \\
\hline $\mathrm{BMI}\left(\mathrm{kg} / \mathrm{m}^{2}\right)^{\dagger}$ & $31 \pm 6.4$ \\
\hline $\mathrm{SES}^{\ddagger}$ & $-3.95(-6.58,-1.55$ \\
\hline \multicolumn{2}{|l|}{ Gender } \\
\hline Men & $148(37.0 \%)$ \\
\hline Women & $252(63.0 \%)$ \\
\hline \multicolumn{2}{|l|}{ Ever smoked } \\
\hline Yes & $169(42.3 \%)$ \\
\hline No & $231(57.8 \%)$ \\
\hline \multicolumn{2}{|l|}{ Current smoker } \\
\hline Yes & $59(14.8 \%)$ \\
\hline No & $341(85.3 \%)$ \\
\hline \multicolumn{2}{|l|}{ Diabetes } \\
\hline Yes & $127(31.8 \%)$ \\
\hline No & $273(68.3 \%)$ \\
\hline \multicolumn{2}{|l|}{ Hypertension } \\
\hline Yes & $288(72.0 \%)$ \\
\hline No & $112(28.0 \%)$ \\
\hline \multicolumn{2}{|l|}{ Dyslipidemia } \\
\hline Yes & $206(51.5 \%)$ \\
\hline No & $194(48.5 \%)$ \\
\hline \multicolumn{2}{|l|}{$\mathrm{PCP}^{\S}$} \\
\hline Yes & $350(87.5 \%)$ \\
\hline No & $50(12.5 \%)$ \\
\hline \multicolumn{2}{|l|}{ Close Contact } \\
\hline Yes & $359(89.8 \%)$ \\
\hline No & $41(10.3 \%)$ \\
\hline Number of phone numbers & $2(2,3)$ \\
\hline Charlson score & $0(0,1)$ \\
\hline \multicolumn{2}{|l|}{ Interpreter used } \\
\hline Yes & $103(25.85)$ \\
\hline No & $297(74.3 \%)$ \\
\hline \multicolumn{2}{|l|}{ Native Language } \\
\hline English & $204(51.0 \%)$ \\
\hline Non-English & $196(49.0 \%)$ \\
\hline \multicolumn{2}{|l|}{ Race } \\
\hline Asian & $18(4.5 \%)$ \\
\hline Black & $143(35.8 \%)$ \\
\hline Hispanic & $215(53.8 \%)$ \\
\hline Multiracial & $6(1.5 \%)$ \\
\hline White & $18(4.5 \%)$ \\
\hline
\end{tabular}

Continued

employment, housing, and education in their ZIP code, normalized to the state average), medical comorbidities (diabetes; obesity; hypertension; dyslipidemia; current or prior smoking; Charlson comorbidity score), health insurance status, having a PCP (defined by current provider listed in the electronic medical record or stated by the participant),
Table 1. Continued

\begin{tabular}{lc}
\hline Characteristic & Values* $^{*}$ \\
\hline Insurance & \\
Commercial & $118(29.5 \%)$ \\
Medicaid & $177(44.3 \%)$ \\
Medicare & $98(24.5 \%)$ \\
Self pay & $7(1.8 \%)$ \\
\hline
\end{tabular}

*Categorical variables are presented as frequencies and percentages. Continuous variables are presented as either means and standard deviations or medians and interquartile ranges, depending on distribution.

${ }^{\dagger} \mathrm{BMI}$, body mass index.

${ }^{\ddagger}$ SES, socioeconomic score. Displayed as a Z score calculated based on the person's address and 6 socioeconomic variables for the neighborhood by zip code (log of median household income; log of median value of housing units; the percentage of households receiving interest, dividend, or net rental income; education; the percentage of adults who completed college; and the percentage of employed individuals in executive, managerial or professional positions) and normalized to the New York State average.

${ }^{\S} \mathrm{PCP}$, primary care physician.

number of phone numbers provided for follow-up, having a close contact, and whether an interpreter was used during enrollment. All modifiable variables (eg, insurance status) were captured at time of enrollment and were not updated over the course of follow-up.

The secondary outcome was defined as the percentage of potential follow-up that was actually achieved. The potential follow-up period was calculated by determining the number of days from each patient's enrollment date to the end date of the study (December 26, 2013). The actual follow-up period was calculated by determining the number of days from the enrollment date until the date the patient was most recently followed-up by electronic medical records or telephone.

\section{Analysis}

To assess association with follow-up, bivariate analyses using $c^{2}$ or Fisher's exact test for categorical variables, and 2-tailed $t$ test or Wilcoxon-MannWhitney $U$ test for continuous variables was performed.

Logistic regression analysis using a stepwise model selection strategy was performed, with a significance of 0.2 required for variable inclusion into the model and a significance of 0.05 to stay in the model. The odds ratio of having successful follow- 
Table 2. Bivariate Analysis of Independent Risk Factors for Loss of Follow-up*

\begin{tabular}{|c|c|c|c|}
\hline Factor & No One-Year Follow-up $(\mathrm{n}=19)$ & Successful One-Year Follow-up $(\mathrm{n}=381)$ & $P$ Value \\
\hline Age (y) & $56 \pm 10$ & $57 \pm 11$ & .7512 \\
\hline $\mathrm{BMI}\left(\mathrm{kg} / \mathrm{m}^{2}\right)^{\dagger}$ & $29 \pm 6.7$ & $31 \pm 6.4$ & .1862 \\
\hline $\mathrm{SES}^{\ddagger}$ & $-3.1371(-6.56945,-1.675089)$ & $-3.9734(-6.6542,-1.647682)$ & .5627 \\
\hline Gender & & & .0532 \\
\hline Men & $11(57.9 \%)$ & $137(36.0 \%)$ & \\
\hline Women & $8(42.1 \%)$ & $244(64.0 \%)$ & \\
\hline Ever smoked & & & .6435 \\
\hline Yes & $9(47.4 \%)$ & $160(42.0 \%)$ & \\
\hline No & $10(52.6 \%)$ & $221(58.0 \%)$ & \\
\hline Current smoker & & & .5025 \\
\hline Yes & $4(21.1 \%)$ & $55(14.4 \%)$ & \\
\hline No & $15(78.9 \%)$ & $326(85.6 \%)$ & \\
\hline Diabetes & & & .4493 \\
\hline Yes & $4(21.1 \%)$ & $123(32.3 \%)$ & \\
\hline No & $15(78.9 \%)$ & $258(67.7 \%)$ & \\
\hline Hypertension & & & .7218 \\
\hline Yes & $13(68.4 \%)$ & $275(72.2 \%)$ & \\
\hline No & $6(31.6 \%)$ & $106(27.8 \%)$ & \\
\hline Dyslipidemia & & & .1902 \\
\hline Yes & $7(36.8 \%)$ & $199(52.2 \%)$ & \\
\hline No & $12(63.2 \%)$ & $182(47.8 \%)$ & \\
\hline $\mathrm{PCP}^{\S}$ & & & .0010 \\
\hline Yes & $13(68.4 \%)$ & $337(88.5 \%)$ & \\
\hline No & $6(31.6 \%)$ & $44(11.5 \%)$ & \\
\hline Close contact & & & .7071 \\
\hline Yes & $18(94.7 \%)$ & $341(89.5 \%)$ & \\
\hline No & $1(5.3 \%)$ & $40(10.5 \%)$ & \\
\hline Number of phone numbers & $2(2,2)$ & $2(2,3)$ & .4856 \\
\hline Charlson score & $0(0,1)$ & $1(0,1)$ & .0480 \\
\hline Interpreter used & & & .2572 \\
\hline Yes & $7(36.8 \%)$ & $96(25.2 \%)$ & \\
\hline No & $12(63.2 \%)$ & $285(74.8 \%)$ & \\
\hline Native Language & & & .0274 \\
\hline English & $5(26.3 \%)$ & $199(52.2 \%)$ & \\
\hline Non-English & $14(73.7 \%)$ & $182(47.8 \%)$ & \\
\hline Race & & & .4468 \\
\hline Asian & $0(0.0 \%)$ & $18(4.7 \%)$ & \\
\hline Black & $4(21.1 \%)$ & $139(36.5 \%)$ & \\
\hline Hispanic & $14(73.7 \%)$ & $201(52.8 \%)$ & \\
\hline Multiracial & $0(0.0 \%)$ & $6(1.6 \%)$ & \\
\hline White & $1(5.3 \%)$ & $17(4.5 \%)$ & \\
\hline Insurance & & & .9068 \\
\hline Commercial & $5(26.3 \%)$ & $113(29.7 \%)$ & \\
\hline Medicaid & $10(52.6 \%)$ & $167(43.8 \%)$ & \\
\hline Medicare & $4(21.1 \%)$ & $94(24.7 \%)$ & \\
\hline Self pay & $0(0.0 \%)$ & $7(1.8 \%)$ & \\
\hline
\end{tabular}

*Categorical variables are presented as frequencies and percentages. Continuous variables are presented as either means and standard deviations or medians and interquartile ranges, depending on distribution.

${ }^{\dagger} \mathrm{BMI}$, body mass index.

${ }^{\ddagger} \mathrm{SES}$, socioeconomic score. Displayed as a $\mathrm{Z}$ score as in the previous table.

${ }^{\S} \mathrm{PCP}$, primary care physician. 
up, given 1 unit increase/category change of the patient factors, was estimated.

With regard to the secondary outcome, the percentage of follow-up completed, separated into tertiles, was the dependent variable. Bivariate analysis for 2 category variables used a 2-tailed $t$ test, and analysis of variance was used for variables with more than 2 categories. Linear regression was used for continuous variables. The degree of follow-up was separated into tertiles for the purpose of an ordered logistic regression analysis, which was applied to variables that were significant or near-significant on bivariate analysis. A $P$ value of $<.05$ was considered significant.

\section{Results}

\section{Follow-up}

At the midpoint of recruitment $(\mathrm{n}=200), 64 \%(63 /$ 99) of patients eligible for 6-month follow-up were reached. By the completion of the study, 95.3\% (381/400) had successful 1-year follow-up. No patients died within 12 months of recruitment.

\section{Group Characteristics}

The average age for cohort was 57 years; $63 \%$ were women, $53.8 \%$ were Hispanic, $35.8 \%$ were black, and $49.0 \%$ had a non-English native language (see Table 1).

Participants who had successful follow-up were significantly more likely to have a PCP $(88.5 \%$ vs $68.4 \%, P<.009)$ and report a native language of English $(52.2 \%$ vs $26.3 \%, P<.027)$ than those lost to follow-up (Table 2). Although specific medical diagnoses were not associated with follow-up, those who had successful follow-up had a higher Charlson comorbidity score (median, 1 vs 0 ; $P<.0480)$. Gender was close to being a statistically significant variable, as women were more likely to achieve follow up $(P=.532)$.

Both PCP and native English language remained significant at logistic regression analysis (Table 3). With native language held constant, having a PCP

Table 3. Logistic Regression Analysis of Factors Associated with Successful Follow-up

\begin{tabular}{llc}
\hline Factor & Odds Ratio (95\% CI) & PValue \\
\hline PCP ${ }^{*}$ Yes & $3.487(1.247-9.753)$ & .0173 \\
Native Language, English & $3.030(1.063-8.621)$ & .0381 \\
\hline
\end{tabular}

*PCP, primary care physician; CI, confidence interval. resulted in 3.5 times the odds of completing the year of follow-up (odds ratio $=3.5$; 95\% CI, 1.25 9.75). Alternatively, with PCP held constant, having a native language of English was associated with 3.0 times the odds of completing the year of followup (odds ratio $=3.0 ; 95 \%$ CI, 1.06-8.62).

Patients who had a PCP were more likely to be in the higher follow-up tertiles $(P=.0046)$ (Table 4). Women had an increased representation in the higher follow-up tertiles than men $(P=.019)$. Although the secondary analysis produced multiple significant variables at the bivariate stage, no variables remained significant for the secondary outcome after logistic regression analysis.

\section{Discussion}

Successful follow-up and clinical trial retention of patients from an urban, predominantly minority, majority-women, poor population is challenging. In the present study, we found that having a PCP made a patient more than 3 times as likely to complete a year of follow-up, with almost nine-tenths of patients with PCPs having successful follow-up compared with just more than two-thirds of patients without a PCP. Having a PCP was also associated with achieving follow-up over a greater proportion of the potential follow-up period (86\% versus $76 \%$ ). Having a PCP may keep a patient more connected with the health care system, thus serving as an important liaison between clinical trial staff and research participants. An implication for research practice is that a patient's PCP status could allow trial designers to focus more resources on maintaining follow-up with participants who have fewer connections to their health care system. An upstream effect of this could be an increased emphasis on connecting patients to PCPs. With regard to current clinical practice, increasing the role of primary care in medicine is an important topic that has ramifications for cost and health outcomes more generally. ${ }^{21}$

It is likely that having a PCP is a proxy for a multitude of factors, including SES, social demands, and personal interest in physical health. Having a PCP may signify higher levels of commitment and flexibility, as well as satisfaction with the health care system. Some studies suggest that health care satisfaction and commitment play a positive role in trial enrollment and retention of minorities. ${ }^{22,23}$ External factors, such as transit options, the local health care 
Table 4. Bivariate Analysis of Independent Variables for Achieving Complete Follow-up*

\begin{tabular}{|c|c|c|c|c|}
\hline Variable & $\%$ Follow up $1^{\text {st }}$ Tertile & $\%$ Follow up $2^{\text {nd }}$ Tertile & $\%$ Follow up $3^{\text {rd }}$ Tertile & $P$ Value \\
\hline Possible Follow-up (days) & $1,359 \pm 378$ & $1,391 \pm 377$ & $1,446 \pm 376$ & .1289 \\
\hline Age (y) & $55 \pm 11.2$ & $60 \pm 10.9$ & $55 \pm 10.9$ & .0016 \\
\hline $\mathrm{BMI}\left(\mathrm{kg} / \mathrm{m}^{2}\right)^{\dagger}$ & $31 \pm 7.2$ & $31 \pm 6.3$ & $30 \pm 5.8$ & .9513 \\
\hline $\mathrm{SES}^{\ddagger}$ & -4.211 & -4.0430 & -4.0625 & .8651 \\
\hline Gender & & & & .0019 \\
\hline Women & $71(53.4 \%)$ & $100(74.1 \%)$ & $81(61.4 \%)$ & \\
\hline Men & $62(46.6 \%)$ & $35(25.9 \%)$ & $51(38.6 \%)$ & \\
\hline Ever smoked & & & & .2245 \\
\hline No & $83(62.4 \%)$ & $73(54.1 \%)$ & $75(56.8 \%)$ & \\
\hline Yes & $50(37.6 \%)$ & $62(45.9 \%)$ & $57(43.2 \%)$ & \\
\hline Current smoker & & & & .6544 \\
\hline No & $115(86.5 \%)$ & $112(83.0 \%)$ & $114(86.4 \%)$ & \\
\hline Yes & $18(13.5 \%)$ & $23(17.0 \%)$ & $18(13.6 \%)$ & \\
\hline Diabetes & & & & .0191 \\
\hline No & $99(74.4 \%)$ & $80(59.3 \%)$ & $94(71.2 \%)$ & \\
\hline Yes & $34(25.6 \%)$ & $55(40.7 \%)$ & $38(28.8 \%)$ & \\
\hline Hypertension & & & & .2592 \\
\hline No & $42(31.6 \%)$ & $31(23.0 \%)$ & $39(29.5 \%)$ & \\
\hline Yes & $91(68.4 \%)$ & $104(77.0 \%)$ & $93(70.5 \%)$ & \\
\hline Dyslipidemia & & & & .0027 \\
\hline No & $69(51.9 \%)$ & $32(30.5 \%)$ & $63(47.7 \%)$ & \\
\hline Yes & $64(48.1 \%)$ & $73(69.5 \%)$ & $69(52.3 \%)$ & \\
\hline $\mathrm{PCP}^{\S}$ & & & & .0046 \\
\hline No & $24(18.0 \%)$ & $7(5.2 \%)$ & $19(14.4 \%)$ & \\
\hline Yes & $109(82.0 \%)$ & $128(94.8 \%)$ & $113(85.6 \%)$ & \\
\hline Close contact & & & & .2400 \\
\hline No & $9(6.8 \%)$ & $15(11.1 \%)$ & $17(12.9 \%)$ & \\
\hline Yes & $124(93.2 \%)$ & $120(88.9 \%)$ & $115(87.1 \%)$ & \\
\hline Number of phone numbers & $2(2,3)$ & $2(2,3)$ & $2(2,3)$ & .3205 \\
\hline Charlson score & $0(0,1)$ & $1(0,2)$ & $1(0,1)$ & .5851 \\
\hline Interpreter used & & & & .9130 \\
\hline No & $97(72.9 \%)$ & $101(74.8 \%)$ & $99(75.0 \%)$ & \\
\hline Yes & $36(27.1 \%)$ & $34(25.2 \%)$ & $33(25.0 \%)$ & \\
\hline Native language & & & & .2019 \\
\hline English & $67(50.4 \%)$ & $62(45.9 \%)$ & $75(56.8 \%)$ & \\
\hline Non-English & $66(49.6 \%)$ & $73(54.1 \%)$ & $57(43.2 \%)$ & \\
\hline Race & & & & .4560 \\
\hline Asian & $4(3.0 \%)$ & $6(4.4 \%)$ & $8(6.1 \%)$ & \\
\hline Black & $49(36.8 \%)$ & $44(32.6 \%)$ & $50(37.9 \%)$ & \\
\hline Hispanic & $72(54.1 \%)$ & $77(57.0 \%)$ & $66(50.0 \%)$ & \\
\hline Multiracial & $2(1.5 \%)$ & $4(29.6 \%)$ & $0(0.0 \%)$ & \\
\hline White & $6(4.5 \%)$ & $4(29.6 \%)$ & $8(6.1 \%)$ & \\
\hline Insurance & & & & .0619 \\
\hline Commercial & $49(36.8 \%)$ & $29(21.5 \%)$ & $40(30.3 \%)$ & \\
\hline Medicaid & $51(38.3 \%)$ & $58(43.0 \%)$ & $68(51.5 \%)$ & \\
\hline Medicare & $29(21.8 \%)$ & $48(35.6 \%)$ & $21(15.9 \%)$ & \\
\hline Self pay & $4(3.0 \%)$ & $0(0.0 \%)$ & $3(2.3 \%)$ & \\
\hline
\end{tabular}

${ }^{*}$ For each bivariate analysis, \% follow-up complete was divided into tertiles. Categorical variables are presented as frequencies and percentages. Continuous variables are presented as either means and standard deviations or medians and interquartile ranges, depending on distribution.

${ }^{\dagger} \mathrm{BMI}$, body mass index.

${ }^{\ddagger} \mathrm{SES}$, socioeconomic score. Displayed as a $\mathrm{Z}$ score as in the previous table.

${ }^{\$} \mathrm{PCP}$, primary care physician. 
infrastructure, and social policies, also play a role. At the individual level, establishing care takes time and, often, money; yet, it is notable that SES score was not a significant predictor of follow-up. It is also notable that having a PCP remained a strong factor when included in a multivariate logistic regression with native language, another significant predictor of follow-up. This suggests that it is not just the ease of communication but rather the process of establishing and maintaining care that is associated with follow-up. When a patient is enrolled in a study, the lack of a PCP indicates that they may be less able to meet the trial's requirements and could require additional support.

Native English speaking was also a significant predictor of successful follow-up. Numerous languages, including Arabic, Bengali, Creole (Haiti), Greek, Khmer (Cambodia), Mende (Sierra Leone), Serbian, Spanish, Tagalog (Philippines), Twi (Ghana), Vietnamese, and Wolof (The Gambia), were preferred by our study patients. Native language may be a proxy for culture, and cultural nuances also play roles in the emphasis on social support, deference to physicians/researchers, and preferential modes of communication. In addition, some of these patients may have been visitors and noncitizens who returned to their country of origin and are, hence, difficult to locate. Some were without regular access to a telephone. In a notable example, 1 trial participant was deaf and preferred follow-up by physical mail correspondence.

Although being a woman did not reach significance as a predictor of follow-up in the primary analysis, women completed a significantly larger percentage of their potential follow-up period in the secondary analysis. Previous studies of retention in communities of racial/ethnic minorities show that men are more likely to be lost. ${ }^{15}$ Gender stereotypes suggest that women are more often caretakers in the home and men may work outside of the home more frequently or for longer hours. In addition, women more commonly present to their PCP for routine care and during illness. ${ }^{24}$ In fact, women represent the majority of people receiving care at our medical center. The relative ease of contacting women research participants is a somewhat unexpected boon for efforts favoring improved trial design to adequately represent women.

We anticipated that 3 variables in particular would be significantly associated with 1-year follow-up: SES score, contact information (both number of phone numbers and having a close contact), and insurance status. Surprisingly, none of these variables were significant. The SES score represents how a patient's ZIP code's income, education, and employment relate to the New York State average. The trial population as a whole was 3 standard deviations below the NY average, ${ }^{20}$ and it may be difficult to detect differences in our uniformly low SES score population. Our observation is consistent with prior research in low-income minority populations. ${ }^{17,25}$

We surmised that contact information would play a role-if a participant provides 1 phone number versus another who gives out 4 , there ought to be a difference in ease of follow-up 12 months later. However, the electronic medical record served as a fallback in our study, providing physician contact information and care updates. Hence, patients who provided fewer phone numbers could achieve follow-up via electronic records. We do not have data to show which particular resources were used to contact each participant for their 12-month followup. The widespread use of cell phones likely mitigates the historic need for multiple phone numbers, as cell phone numbers typically remain constant. Similar to our need for various methods of contact, having multiple methods to contact participants and collect data has been discussed as a potential avenue of increasing retention in trials. ${ }^{25} \mathrm{~A}$ recent assessment of retention strategies in the United Kingdom revealed a similar line of thinking, with various methods used, including contacting PCPs and SMS text messaging. ${ }^{26}$ The effectiveness of phone calls to participants is uncertain, as 1 recent study showed that regular phone calls from a coordinating center did not improve retention. ${ }^{27}$ Our study did not use phone calls as a variable; yet, this offers an interesting contrast.

We anticipated that patients who did not have insurance would be less likely to receive follow-up. These participants have fewer resources, so a lack of time and money could result in difficulty communicating. However, we did not find insurance status to be significant. Patients with Medicaid comprised a nonsignificantly greater percentage of the group that did not achieve follow-up.

We expected that patients with a 1-year potential follow-up duration would more readily achieve complete follow-up than those with a potential follow-up duration of 5 years. This was not the case, 
suggesting that the follow-up period is not as important as the characteristics of the population. This supports the feasibility of trials with longer follow-up periods.

\section{Limitations}

The moderate-sized cohort $(n=400)$ is a limitation of this study. In particular, the group that did not receive follow-up totaled only 19 patients. Several variables had small subgroups. Despite the limitation in size, the relationship between having a PCP and being a native English speaker were statistically significantly related to successful follow-up and are likely to be clinically meaningful. A larger study would be beneficial in terms of adding multiple centers with different encatchments, as this trial was performed at a single site with a single, albeit diverse, patient mix. The population that was studied (minority, low SES, majority-women) varies considerably from the demographics of many areas. Potential interventions to improve follow up were not employed in this study, nor did we look at whether patients with PCPs might respond differently to said interventions than patients without PCPs. We were reliant on participants informing us of their use of outside doctors and hospitals; not having consistent access to participants' entire medical networks was another constraint. In addition, there was no third-party verification of whether participants had a PCP; the reliance on electronic medical records and self-reporting of PCP status is an important limitation. Although the variable of having a PCP was statistically significant, PCPs were also themselves contacted to assist with follow-up, resulting in possible confounding variables. However, the various phone numbers and close contacts were used for follow-up and neither was statistically significant. Therefore, we suggest that methods of contacting patients did not impact follow-up as much as inherent patient characteristics.

\section{Future Directions}

In conclusion, we found that successful follow-up was associated with patients having a PCP and being native English speakers. When designing clinical trials, especially in an urban, culturally diverse, underserved environment, it is vital to assess whether patients are connected to the health care system and that their barriers to care, both internal and external, are addressed. Most importantly, exploring whether participants have a PCP should allow researchers to customize follow-up protocols. Other work has recently asked whether routine clinical care could improve retention, ${ }^{27}$ and indeed, this question warrants additional study. Successful follow-up depends on an awareness of a patient's individual needs and communication preferences. It is also important that language and cultural differences are considered. This can be difficult in the fast-paced environment of medical data gathering, but the rigor and generalizability of outcomes research is at stake.

To see this article online, please go to: http://jabfm.org/content/ 33/3/431.full.

\section{References}

1. Lord SJ, Irwig L, Simes RJ. When is measuring sensitivity and specificity sufficient to evaluate a diagnostic test, and when do we need randomized trials? Ann Intern Med 2006;144:850-5.

2. Lauer MS. Comparative effectiveness research: the view from the NHLBI. J Am Coll Cardiol 2009;53: 1084-6.

3. Akl EA, Briel M, You JJ, et al. Potential impact on estimated treatment effects of information lost to follow-up in randomised controlled trials (LOSTIT): systematic review. BMJ 2012;344:e2809e2809.

4. Altman DG. Missing outcomes in randomized trials: addressing the dilemma. Open Med 2009;3: e51-3.

5. Fleming TR. Addressing missing data in clinical trials. Ann Intern Med 2011;154:113-7.

6. Bower P, Brueton V, Gamble C, et al. Interventions to improve recruitment and retention in clinical trials: a survey and workshop to assess current practice and future priorities. Trials 2014;15: 399.

7. Coakley M, Fadiran EO, Parrish LJ, Griffith RA, Weiss E, Carter C. Dialogues on diversifying clinical trials: successful strategies for engaging women and minorities in clinical trials. J Womens Health (Larchmt) 2012;21:713-6.

8. Zhang T, Tsang W, Wijeysundera HC, Ko DT. Reporting and representation of ethnic minorities in cardiovascular trials: a systematic review. Am Heart J 2013;166:52-7.

9. Paskett ED, Reeves KW, McLaughlin JM, et al. Recruitment of minority and underserved populations in the United States: the Centers for Population Health and Health Disparities experience. Contemp Clin Trials 2008;29:847-61.

10. Davis AM, Vinci LM, Okwuosa TM, Chase AR, Huang ES. Cardiovascular health disparities: a systematic review of health care interventions. Med Care Res Rev 2007;64:29S-100S. 
11. Pilote L, Dasgupta K, Guru V, et al. A comprehensive view of sex-specific issues related to cardiovascular disease. CMAJ 2007;176:S1-44.

12. Harris DJ, Douglas PS. Enrollment of women in cardiovascular clinical trials funded by the National Heart, Lung, and Blood Institute. N Engl J Med 2000;343:475-80.

13. Ghare M, Chandrasekhar J, Mehran R, et al. Sex disparities in cardiovascular device evaluations: strategies for recruitment and retention of female patients in clinical device trials. JACC Cardiovasc Interv 2019;12:301-8.

14. National Institutes of Health. Inclusion of women and minorities as participants in research involving human subjects. Available from: https://grants.nih. gov/grants/funding/women_min/women_min.htm.

15. Warner ET, Glasgow RE, Emmons KM, et al. Recruitment and retention of participants in a pragmatic randomized intervention trial at three community health clinics: results and lessons learned. BMC Public Health 2013;13:192.

16. Senturia YD, McNiff Mortimer K, Baker D, et al. Successful techniques for retention of study participants in an inner-city population. Control Clin Trials 1998;19:544-54.

17. Nicholson LM, Schwirian PM, Klein EG, et al. Recruitment and retention strategies in longitudinal clinical studies with low-income populations. Contemp Clin Trials 2011;32:353-62.

18. Yancey AK, Ortega AN, Kumanyika SK. Effective recruitment and retention of minority research participants. Annu Rev Public Health 2006;27:1-28.

19. Levsky JM, Travin MI, Spevack DM, et al. Rationale and design of a randomized controlled trial comparing stress myocardial perfusion imaging with coronary CT angiography as the initial imaging study for intermediate-risk patients admitted with chest pain. J Cardiovasc Comput Tomogr 2009;3:264-71.

20. Levsky JM, Spevack DM, Travin MI, et al. Coronary computed tomography versus radionuclide myocardial perfusion imaging in chest pain patients admitted to telemetry: a randomized, controlled trial. Ann Intern Med 2015;163:174-83.

21. Bazemore A, Petterson S, Peterson L, et al. Higher primary care physician continuity is associated with lower costs and hospitalizations. Ann Fam Med 2018;16:492-7.

22. Gadegbeku CA, Stillman PK, Huffman MD, Jackson JS, Kusek JW, Jamerson KA. Factors associated with enrollment of african americans into a clinical trial: results from the african american study of kidney disease and hypertension. Contemp Clin Trials 2008;29:837-42.

23. Janson SL, Alloto ME, Boushey HA; Asthma Clinical Trials Network. Attrition and retention of ethnically diverse subjects in a multicenter randomized controlled research trial. Control Clin Trials 2001;22:236S-43S.

24. Bertakis KD, Azari R, Helms L, Callahan E, Robbins J. Gender differences in the utilization of health care services. J Fam Pract 2000;49:147-52.

25. Nabil-Khorazaty MN, Johnson AA, Kiely M, et al. Recruitment and retention of low-income minority women in a behavioral intervention to reduce smoking, depression, and intimate partner violence during pregnancy. BMC Public Health 2007;7:233.

26. Kearney A, Daykin A, Shaw ARG, et al. Identifying research priorities for effective retention strategies in clinical trials. Trials 2017;18:406.

27. Glassman AB, Stockdale CR, et al. Effect of telephone calls from a centralized coordinating center on participant retention in a randomized clinical trial. Clin Trials. In press.

28. Brunsdon D, Biesty L, Brocklehurst P, et al. What are the most important unanswered research questions in trial retention? A James Lind Alliance Priority Setting Partnership: the PRioRiTy II (Prioritising Retention in Randomised Trials) study. Trials 2019;20:593. 